\title{
TECHNOLOGY THAT TOUCHES LIVES: TELECONSULTATION TO BENEFIT PERSONS WITH UPPER LIMB LOSS
}

\author{
LYNSAY R. WHELAN, OTR/L, NATHAN WAGNER, CP, OTR/L \\ TOUCH BIONICS, HILLIARD, OH
}

\section{ABSTRACT}

While over 1.5 million individuals are living with limb loss in the United States (Ziegler-Graham et al., 2008), only 10\% of these individuals have a loss that affects an upper limb. Coincident with the relatively low incidence of upper limb loss, is a shortage of the community-based prosthetic rehabilitation experts that can help prosthetic users to more fully integrate their devices into their daily routines. This article describes how expert prosthetists and occupational therapists at Touch Bionics, a manufacturer of advanced upper limb prosthetic devices, employ Voice over the Internet Protocol (VolP) videoconferencing software telehealth technologies to engage in remote consultation with users of prosthetic devices and/or their local practitioners. The Touch Bionics staff provide follow-up expertise to local prosthetists, occupational therapists, and other health professionals. Contrasted with prior telephone-based consultations, the video-enabled approach provides enhanced capabilities to benefit persons with upper limb loss. Currently, the opportunities for Touch Bionics occupational therapists to fully engage in patient-based services delivered through telehealth technologies are significantly reduced by their need to obtain and maintain professional licenses in multiple states.

Keywords: Upper limb loss, upper limb prosthetic devices, telehealth, telerehabilitation, occupational therapy

\section{INTRODUCTION}

It is estimated that over 1.5 million individuals are living with limb loss in the United States (Ziegler-Graham et al., 2008). Approximately $10 \%$ of these individuals have loss affecting an upper limb(s), with only 2,000 new cases proximal to the wrist each year (Dillingham et al., 2002). Because the percentage of upper limb amputees is small in comparison to all individuals with limb loss, and these individuals are geographically dispersed, many occupational therapists and prosthetists have little experience treating persons with upper limb loss.

Touch Bionics, a manufacturer of advanced upper limb prosthetic devices, supports patient fittings with customized prosthetic devices. A typical fitting requires many hours of intense rehabilitation. After initial training, many patients require additional therapy and support before they can fully integrate the devices into daily routines. Recognizing this need, Touch Bionics staff members provide follow-up expertise to the local prosthetists, occupational therapists, and the other allied health professionals who work with patients following their acquisition of the prosthetic devices. Though the telephone can be used to answer simple questions, inperson consultations represent an ideal way to educate, train, and assist local clinicians. Unfortunately, in-person assistance is not always possible, feasible, or timely due to many of these patients' distant geographic locations.

\section{TOUCH BIONICS' USE OF TELEHEALTH}

Recognizing that in-person consultations are not possible for many patients, Touch Bionics began to incorporate telehealth technologies as an alternative model to educate, train, and assist clinicians and their patients. The use of Voice over the Internet Protocol (VolP) videoconferencing software (e.g., Skype, TeamViewer, GoToMeeting) allows a distant prosthetist and/or occupational therapist to remotely engage in a session with a Touch Bionics prosthetist or occupational therapist. The Touch Bionics staff selected VoIP software because many professionals and patients already use or have experience with this technology. With a webcam located at each location, the communication and collaboration can progress well beyond what might be accomplished via a voice-only phone call. The prosthetist, or occupational therapist, along with their patient, can point to areas being described, demonstrate problems or questions, and get detailed visual directions from the remote prosthetist or occupational therapist.

The software that enables remote access to the local prosthetists' or therapists' computer is especially beneficial. Computer sharing software with video conferencing capability and remote access to the local clinician's computer screen allows the Touch Bionics prosthetist or occupational therapist to remotely evaluate 
and adjust the patient's prosthetic device even though they are miles away. The ability to see information transmitted from the processor in the prosthesis (i.e., connected to the computer through Bluetooth technology from a remote location) facilitates troubleshooting and enables software adjustments that address the patients' needs.

Building upon the successes of telehealth for remote consultations between prosthetists and persons fitted with Touch Bionics devices, occupational therapists at Touch Bionics recognized the opportunity to provide similar tele-support to community-based occupational therapists, thereby increasing the likelihood that persons with upper limb loss will fully integrate their new prostheses into their daily routines.

Occupational therapists, by virtue of their skill sets and experiences, are particularly well equipped to assess whether patients are optimally using their prostheses to engage in activities of daily living (i.e. dressing, eating, bathing, grooming) and instrumental activities of daily living (i.e. care of others, financial, health, and home management), education, work, play, leisure, and social participation. Performance enhancement requires both task analysis and task modification, with the end goal that patients will fully integrate their devices into their daily routines. The need for rehabilitative services, including consultation for task modification, is common as patients return home and encounter unexpected challenges. For example, when using a prosthetic device to cut meat, the use of the fork to stabilize the meat tends to be most effective when the tines of the fork are faced downward and the fork is held with lateral prehension. This is not the typical way to cut meat, but a therapist guiding a patient in this task modification would enhance the patient's performance and this intervention could be justified as billable event. Similarly, an individual returning to work may benefit from consultation with an occupational therapist to acquire strategies and task modifications that facilitate integration of the individual's prosthetic device into job tasks, ultimately enhancing their job performance. In each case, the patient would benefit from a consultation with an occupational therapist with expertise in upper limb prosthetics, to identify solutions to the challenges encountered. Additionally, individuals who are not actively receiving rehabilitation services may encounter an isolated problem, and a one-time telehealth consultation may meet their needs.

Pre- and post- prosthetic training and the technology can be intimidating to occupational therapists who have never worked with this population or with hightech prosthetic devices. Telehealth bridges this gap in experience, affording the opportunity for remote consultations to occur between therapists, so that their patients experience a smooth transition to follow-up care in their own community.

\section{ADAPTATIONS TO LICENSURE BARRIERS}

Initially, Touch Bionics occupational therapists were licensed in the three states, (i.e., Ohio, Georgia, and California) that house the three main company offices.

Unfortunately, the current licensure model does not enable Touch Bionics therapists to consult with patients from across the country who contact the company directly. It is a time consuming and costly process to obtain multiple licenses for the therapists, and maintaining the licenses and abiding by the various state's regulations and continuing education requirements requires much effort. The ability to provide follow-up therapeutic services directly to patients in their home using telehealth would require therapists to obtain and maintain licensure in each state where the patients are located, an expensive and time-consuming barrier.

Instead, Touch Bionics therapists provide expert consultations without charge to local therapists who provide the direct care for the patients. The services are educational in nature and support the local therapist, who is the licensed provider working directly with the patient. In this model, the local therapist conducts the rehabilitation and bills for the therapy -- supported by consultation with a Touch Bionics therapist with expertise with prosthetics.

Though Touch Bionics takes a financial loss by providing follow-up educational services without charge to local therapists, the educational consultations through telehealth technologies enable patients to achieve better outcomes. The resultant enhanced rehabilitation outcomes mutually benefit the patient, the local therapist, the company, and society. 


\section{CONCLUSION}

In conclusion, individuals with upper limb loss constitute an underserved population. The use of electronic communication technology allows for enhanced care to this population. While Touch Bionics has successfully incorporated telehealth technologies into some aspects of patient care, there are many instances where patients could further benefit from increased access to specialists through the use of telerehabilitation. Licensure models that promote interstate license portability would greatly benefit this underserved population.

\section{REFERENCES:}

1. Dillingham, T. R., Pezzin, L. E., \& MacKenzie, E. J. (2002). Limb amputation and limb deficiency: Epidemiology and recent trends in the United States. Southern Medical Journal, 95, 875-883.

2. Ziegler-Graham, K., MacKenzie, E.J., Ephraim, P.L., Travison, T.G., \& Brookmeyer, R. (2008). Estimating the prevalence of limb loss in the United States - 2005 to 2050. Archives of Physical Medicine and Rehabilitation, $89,422-429$.

\section{EDITOR'S NOTE:}

Current medical and legal practices dictate that it is the location of the client that determines the state in which the practitioner must be licensed. Some states do have consultation and licensure exemption provisions, though largely untested for services delivered through telehealth technologies. It is the practitioners' responsibility to know their scope of practice laws and regulations for the states in which they render services, including services delivered remotely through telehealth technologies. Practitioners should contact state professional licensure boards for further clarification on policies related to consultation, licensure exemption provisions, and acceptable models of service delivery prior to rendering services. 
